\title{
Quantifying Clustering in Disordered Carbon Thin Films
}

\author{
J. D. Carey $^{\text {a) }}$ and S. R. P. Silva \\ Advanced Technology Institute, School of Electronics and Physical Sciences, \\ University of Surrey, Guildford, GU2 7XH, United Kingdom
}

\begin{abstract}
The quantification of disorder and the effects of clustering in the $\mathrm{sp}^{2}$ phase of amorphous carbon thin films are discussed. The $\mathrm{sp}^{2}$ phase is described in terms of disordered nanometer sized conductive $\mathrm{sp}^{2}$ clusters embedded in a less conductive $\mathrm{sp}^{3}$ matrix. Quantification of the clustering of the $\mathrm{sp}^{2}$ phase is estimated from optical as well as from electron and nuclear magnetic resonance methods. Unlike in other disordered group IV thin film semiconductors, we show that care must be exercised in attributing a meaning to the Urbach energy extracted from absorption measurements in the disordered carbon system. The influence of structural disorder, associated with $\mathrm{sp}^{2}$ clusters of similar size, and topological disorder due to undistorted clusters of different sizes is also discussed. Extensions of this description to other systems are also presented.
\end{abstract}

a) Electronic mail: David.Carey@surrey.ac.uk 


\section{Introduction}

Understanding the effects of disorder on the electronic properties of amorphous carbon $(a-\mathrm{C})$ is important for the potential development of active devices. The presence of both $\mathrm{sp}^{2}$ and $\mathrm{sp}^{3}$ bonding results in a mixed phase material where many of the electronic, transport and optical properties are determined by the $\mathrm{sp}^{2}$ phase with the mechanical and tribological properties being largely determined by the $\mathrm{sp}^{3}$ phase $[1,2]$. The ability to deposit $a-\mathrm{C}$ films over large areas with mirror smooth surfaces has resulted in the use of these films in biomedical coatings and in hard disks, however, only limited success on the development of electronic devices has emerged [3]. One notable and recent success has been the report of resonant tunnelling and fast switching from an all carbon quantum well structure with the barrier and well materials containing differing amounts of $\mathrm{sp}^{2}$ and $\mathrm{sp}^{3}$ carbon [4]. In general, the $\mathrm{sp}^{2}$ phase in $a-\mathrm{C}$ and its hydrogenated form, $a-\mathrm{C}: \mathrm{H}$, tends to exist as nm-sized clusters in the form of graphitic rings or, to a lesser extent, olephinic chains. These clusters of $\operatorname{sp}^{2} \mathrm{C}$ atoms give rise to $\pi$ and $\pi^{*}$ bands and many of the optical properties are discussed in terms of transitions between the occupied $\pi$ states to the unoccupied $\pi^{*}$ states. The $\mathrm{sp}^{3}$ phase is associated with the $\sigma$ and $\sigma^{*}$ states which lie further separated from each other.

In most disordered semiconductors, the optical energy gap between extended states is called the Tauc band gap. In the absence of disorder, the Tauc gap is given by the separation between the $\pi-\pi^{*}$ bands and the larger the size of the $\mathrm{sp}^{2}$ cluster the smaller the Tauc gap. An alternative band gap that is sometimes employed is to measure the energy at which the absorption coefficient is $10^{4} \mathrm{~cm}^{-1}$; this is known as the $\mathrm{E}_{04}$ gap. Values associated with the $\mathrm{E}_{04}$ gap tend to be larger than the Tauc gap as the absorption associated with $10^{4} \mathrm{~cm}^{-1}$ tends to occur between states beyond the $\pi$ and $\pi *$ band edges. Transitions between extended-tolocalised states in amorphous semiconductors are usually characterised by the Urbach energy, $\mathrm{E}_{\mathrm{u}}$. In this way the Urbach energy is often regarded as a measure of disorder within the film and estimates of the Urbach energy can be found by fitting an exponential function to the slope 
of the absorption edge. A second measure of disorder, based upon bond angle distortion, is the full width half maximum of the Raman active $G$ band, $\Gamma_{\mathrm{G}}$. This Raman signal is found at around $1580 \mathrm{~cm}^{-1}$ and originates from the $\mathrm{E}_{2 \mathrm{~g}}$ vibration between $\mathrm{sp}^{2} \mathrm{C}$ bonds and is therefore present in all $\mathrm{sp}^{2} \mathrm{C}$ containing materials [5]. It should be noted that special care must be exercised when using single wavelength Raman measurements and there is now a growing realisation that multi-wavelength measurements are important as a measure of disorder and clustering in $a$-C thin films. For example, if two films have the same $\mathrm{G}$ peak position under a single visible wavelength Raman measurement, the sample with the lower G peak shift under UV Raman excitation will have the higher degree of clustering [5]. A third measure of disorder is the dispersion of $\mathrm{G}$ peak position with excitation wavelength. Any dispersion of the $\mathrm{G}$ peak arises from the resonant excitation of $\mathrm{sp}^{2}$ clusters of different sizes at different excitation wavelengths. As a consequence of this, films that consist of a fully delocalised $\mathrm{sp}^{2}$ phase e.g. graphite, will not show any $\mathrm{G}$ peak dispersion. It is the aim of this paper to discuss the quantification of the disorder found in amorphous carbon materials. Specifically, we will examine what information can be obtained by optical methods as well as probing electron states near the Fermi level using magnetic resonance methods.

\section{Disorder in Amorphous Carbon - Optical Measurements}

For mixed phase materials with different sizes of $\mathrm{sp}^{2}$ clusters, dispersion of the $\mathrm{G}$ band will occur, as shown in Fig. 1 (a). Since the dispersion of the G peak reflects the presence of different sized clusters, it also acts as a measure of the Tauc band gap. The three series of data in Fig. 1(a) are for a high $\mathrm{sp}^{2}$ containing narrow Tauc gap graphitic carbon film, with a dispersion of only $0.09 \mathrm{~cm}^{-1} / \mathrm{nm}$, a diamond-like carbon film with a dispersion of $0.23 \mathrm{~cm}^{-1} / \mathrm{nm}$ and a wide gap polymer-like film with a dispersion of $0.31 \mathrm{~cm}^{-1} / \mathrm{nm}$. This result shows that the as the Tauc gap increases the amount of $\mathrm{G}$ peak dispersion also increases. Indeed, Casiraghi et al. have shown that the dispersion of the $\mathrm{G}$ band also apparently increases linearly with Tauc 
gap [5]. For a film with a Tauc gap of $1.5 \mathrm{eV}$, they estimated a dispersion is $0.22 \mathrm{~cm}^{-1} / \mathrm{nm}$, rising to $0.35 \mathrm{~cm}^{-1} / \mathrm{nm}$ for a film with a $3 \mathrm{eV}$ Tauc gap. Such combined optical $[6,7,8]$ and Raman studies [5, 9] are useful at probing qualitatively the disorder present, however have also produced some potentially unusual results. Shown in Fig. 1(b) is the variation of Urbach energy, $\mathrm{E}_{\mathrm{u}}$, with Tauc gap for several series of $a-\mathrm{C}$ and $a-\mathrm{C}: \mathrm{H}$ films where it can be seen that the Urbach energy increases with Tauc gap. Specifically any description of disorder should explain why larger values of $E_{u}$ are observed at the highest Tauc gaps (Fig. 1 (b)) where the cluster sizes are smallest and as a result there is only a limited number of different cluster conformations possible. In addition, it has also been observed that there is a maximum in the $\mathrm{G}$ band line width with Tauc gap and that the $G$ band line width depends on the excitation wavelength with the narrower values of the linewidth observed at increasing excitation energy [10]. This observation of dispersion of the Raman line width implies that there is an inhomogeneous distribution in bond angle disorder. Furthermore, the reduction of the $\mathrm{G}$ width with increasing excitation energy implies that there is a narrower distribution of higher gap $\mathrm{sp}^{2}$ states. This conclusion is clearly in contradiction with the suggestion above that there must be a larger distribution of $\mathrm{sp}^{2}$ states required for the large Urbach energy.

Whilst the Urbach energy may be a useful measure of disorder in other amorphous group IV semiconductors such as $a$-Si and $a$-Ge, the larger number of bonding configurations in the mixed phase carbon system makes its use as a measure of disorder more questionable. Indeed, in a recent paper examining the optical properties of hydrogenated amorphous silicon carbon alloys, Nguyen-Tran and colleagues showed that $\mathrm{E}_{\mathrm{u}}$ increased with methane flow (i.e. carbon content and wider Tauc gap) and also concluded that the Urbach energy may not be a good of disorder [11]. From the discussion above, it is apparent that the various optical measurements that have been untaken are at worst producing inconsistent results or that they are measuring different types of disorder present. Fanchini and Tagliaferro proposed that disorder can be sub-divided into two types [12]: 
(i) a structural disorder associated with clusters of the same size but with different amounts of distortion and

(ii) a topological disorder arising from undistorted clusters but of different sizes.

The former type of disorder would increase both the Urbach energy and the G band linewidth, whereas the latter would increase the Urbach energy but have no effect on the G linewidth.

\section{Disorder in Amorphous Carbon - Magnetic Resonance Measurements}

It is therefore apparent that the extent of distortion in the $\mathrm{sp}^{2}$ clusters plays an important role. As a consequence the difference between distorted and undistorted $\mathrm{sp}^{2}$ clusters needs to be explored. The location of the $\mathrm{sp}^{2}$ clusters in the energy gap depends on two factors; whether they consist of even or odd numbers of carbon atoms and whether they are distorted. Undistorted even numbered clusters will give rise to states near the Fermi level $\left(E_{\mathrm{F}}\right)$ only if they are sufficiently large. This differs from the case of odd numbered clusters which can give rise to gap states even if composed of a small number of atoms. In general, distorted clusters gives rise to states that are closer to $E_{\mathrm{F}}$ than undistorted clusters. Electron spin resonance (ESR) - also called electron paramagnetic resonance, is a technique that can be used to monitor singly occupied electron states near the Fermi level [13]. In the single electron approximation the density of states will be filled pairwise by electrons in order of the orbital energy and within this approximation no ESR signals should be visible. In order to explain the observations of the ESR signal observed in many different forms of $a$-C, correlation effects which prevent the spin pairing from occurring need to be considered. The correlation energy, $U$, is the difference between the Coulomb repulsion energy of two electrons in the same orbital and the energy gained by lattice relaxation at the defect site when the second electron is added to the orbital. Positive and negative $U$ centers have different properties. If $U$ is positive all the defect states will be single occupied and the sample will exhibit paramagnetism where states from $E_{\mathrm{F}}-U$ to $E_{\mathrm{F}}$ are singly occupied; states below $E_{\mathrm{F}}-U$ are doubly occupied and those above $E_{\mathrm{F}}$ are empty. 
In this manner ESR can give a measure of the density of $\mathrm{sp}^{2}$ states at the Fermi level, provided that a majority of these states have a net unpaired electron spin present. Note that measurements of ESR spin densities do not themselves measure the $\mathrm{sp}^{2}$ content. For example, electron energy loss spectroscopy measurements made on PECVD grown films showed that the $\pi$ bonds per carbon atom, taken to be the $\mathrm{sp}^{2}$ content, varied from $63 \%$ to $72 \%$ for a change of negative self bias from $100 \mathrm{~V}$ to $265 \mathrm{~V}$. This was accompanied by an increase in the spin density from $4.9 \times 10^{19} \mathrm{~cm}^{-3}$ to $1.5 \times 10^{20} \mathrm{~cm}^{-3}$. However, whilst increasing the negative self bias to $540 \mathrm{~V}$ the spin density hardly changed $\left(2-3 \times 10^{20} \mathrm{~cm}^{-3}\right)$, the $\mathrm{sp}^{2}$ content increased to nearly $100 \%$. [13] Films grown with a high $\mathrm{H}$ content and low internal stresses tend to have much lower defect densities typically $\sim 10^{17} \mathrm{~cm}^{-3}[14]$.

Films of $a-\mathrm{C}: \mathrm{H}$ were deposited on either the driven or earthed electrode of a Plasma Technology DP800 capacitively coupled radio-frequency $13.56 \mathrm{MHz}$ plasma enhanced chemical vapor deposition system. Films were grown on Corning 7059 glass for optical measurements and high-resistivity substrates for ESR measurements. During deposition, feed gases of methane $(30 \mathrm{sccm})$ with $\mathrm{He}(75 \mathrm{sccm})$ were used and the total deposition pressure was 200 mTorr. Film thicknesses were measured using ellipsometry. Figure 2 shows the variation of the spin density, $\mathrm{N}_{\mathrm{s}}$, and peak-to-peak linewidth, $\Delta \mathrm{B}_{\mathrm{pp}}$, with negative self-bias for a series of films deposited from $\mathrm{CH}_{4}$ as a hydrocarbon source gas. Two other sets reported in the literature are also presented $[7,8]$. It is evident that the spin density rises from a low value of $\sim 10^{17} \mathrm{~cm}^{-3}$ and saturates at $10^{20} \mathrm{~cm}^{-3}$. The ESR linewidth initially increases, often as the spin density increases, but at higher biases the linewidth decreases before saturating. Since the spin densities under high bias conditions reported are typically $\sim 10^{20} \mathrm{~cm}^{-3}$, corresponding to $\sim 1$ at. $\%$ of the material present, we may reasonably assume that the ESR signal can be considered as originating from a dilute paramagnetic material. It is therefore possible to apply the theory of Abragam to calculate the contribution to $\Delta \mathrm{B}_{\mathrm{pp}}$ from the dipole-dipole interaction between like spins [15]. Such a broadening mechanism will produce a Lorentzian ESR lineshape, consistent 
with the observed lineshape. At lower biases, the lineshape is often Gaussian, characteristic of inhomogeneous broadening, and usually attributed to unresolved $\mathrm{H}$ hyperfine interactions. Assuming a spin concentration $\mathrm{N}_{\mathrm{s}}$, measured in $\mathrm{cm}^{-3}$, the contribution to the dipolar interaction $\Delta \mathrm{B}_{\mathrm{pp}}$, measured in $\mathrm{mT}$, can be given as [13]

$$
\Delta \mathrm{B}_{p p}=8.12 \times 10^{-21} N_{S}
$$

Figure 3 shows the variation of $\Delta B_{p p}$ against $N_{S}$ directly for the same three series of data reported in Fig. 2, as well the predicted linewidth (dashed line) based solely on dipolar broadening using Eq. (1). It is apparent that significant reductions in $\Delta \mathrm{B}_{\mathrm{pp}}$ are occurring at high $\mathrm{N}_{S}$, when compared with the values predicted by Eq. (1), indicating an alternative mechanism to dipolar broadening is responsible for the linewidth. This additional interaction is due to the motional averaging of the electron wavefunction within the $\mathrm{sp}^{2}$ cluster. As the $\mathrm{sp}^{2}$ cluster increases in size the probability of the electron being associated with a particular atom decreases. This spread out or delocalization of the wavefunction as the cluster size increases is accompanied by a general reduction in the Tauc gap. Larger clusters will have greater amount of delocalization and the smaller Tauc gap.

It is further possible to relate the extent of the clustering by combining the ESR results above with the conclusions from nuclear magnetic resonance measurements (NMR). Jäger et al. used cross polarisation ${ }^{13} \mathrm{C}$ NMR to measure the effects on the proton relaxation times in different environments of films deposited from acetylene and benzene. It was reported [16] that two proton spin-lattice relaxations time constants of 14 and $120 \mathrm{~ms}$ were present and were attributed to the presence of two differently relaxing proton systems. The shorter relaxation time originated from $\mathrm{CH}$ groups in the $\mathrm{sp}^{2}$ and $\mathrm{sp}^{3}$ matrix. The longer relaxation time was believed to originate from the short $\mathrm{CH}_{2}$ polymer units with a $\mathrm{sp}^{3}$ configuration. These two environments are separated by regions of non-hydrogenated $\mathrm{sp}^{2} \mathrm{C}$. It was also reported that the shorter $\mathrm{T}_{1}$ component varied little with temperature suggesting that the protons are being relaxed via spin 
diffusion to paramagnetic centers. Therefore it is the degree of electron aggregation or clustering around these paramagnetic centers that determines the proton relaxation.

On the basis of NMR measurements, Tamor et al. estimated the protonated and nonprotonated $\mathrm{C}$ concentration with either $\mathrm{sp}^{2}$ or $\mathrm{sp}^{3}$ hybridisation [17]. At a self-bias of $-500 \mathrm{~V}$, the $\mathrm{H}$ content was estimated to be 36 at. $\%$ and the fraction of protonated $\mathrm{sp}^{3} \mathrm{C}$ atoms to be 0.16 , non-protonated $\mathrm{sp}^{3} \mathrm{C}$ atoms to be 0.20 , protonated $\mathrm{sp}^{2} \mathrm{C}$ atoms to be 0.25 , and nonprotonated $\mathrm{sp}^{2} \mathrm{C}$ atoms to be 0.37 . For a film density of $1.7 \mathrm{~g} \mathrm{~cm}^{-3}$, this result suggests there are $5.1 \times 10^{22} \mathrm{sp}^{2} \mathrm{C}$ atoms $\mathrm{cm}^{-3}$ in the film. If the signal observed from ESR presented in Fig. 2(a) is associated with $\mathrm{sp}^{2} \mathrm{C}$ centers, then for a typical value of $\mathrm{N}_{\mathrm{s}}$ of $10^{20} \mathrm{~cm}^{-3}$, this corresponds to 1 spin per $510 \mathrm{sp}^{2} \mathrm{C}$ atoms or 1 spin per 300 atoms of non-protonated $\mathrm{sp}^{2} \mathrm{C}$ atoms. If these spins were randomly distributed throughout the film co-operative effects, such as exchange, should not occur. Since exchange effects are observed to films grown at high bias, the combined use of NMR and ESR confirms and quantifies the clustering of the $\mathrm{sp}^{2}$ phase that has taken place.

\section{Conclusions}

In conclusion, the disorder and clustering within a wide variety of amorphous carbon thin films can be characterized in terms of states near the Fermi level. For polymer-like films with small clusters and a small distribution of cluster sizes the Urbach energy is not a good measure of disorder and topological disorder dominates. In DLC films, the increase in spin density $\left(10^{20} \mathrm{~cm}^{-3}\right)$ coupled with the increase in the $\mathrm{G}$ band width implies more distorted clusters are present. At the higher spin density the ESR linewidth is no longer determined by the dipole interaction, with intracluster exchange interactions determining the linewidth. In this regime both topological and structural disorder is present and can affect the stress in the film.

The authors would like to thank the EPSRC for funding via an Advanced Research Fellowship (JDC) and via a Portfolio Partnership Award and the Carbon Based Electronics programme. 


\section{References}

1 S. R. P. Silva, J. D. Carey, R. U. A. Khan, E. G. Gerstner and J. V. Anguita, 'Amorphous carbon thin films', chapter 9, volume 4, Handbook of Thin Film Materials, Ed H.S. Nalwa, (Academic Press, New York, 2002) pp.403 -506.

2 J. Robertson, Mater. Sci. Eng. R. 37129 (2002).

3 S.R.P. Silva and J.D. Carey Diam. Relat. Mater. 12, 151 (2003).

4 S. Bhattacharyya, S.J. Henley, E. Mendoza, L. Gomez-Rojas and S.R.P. Silva, Nature Mat. 5, 19 (2006).

5 C. Casiraghi, A.C. Ferrari and J. Robertson, Phys. Rev. B 72, 085401 (2005).

6 J. Ristein, J. Schäfer and L. Ley, Diam. Relat. Mater. 4, 509 (1995).

7 A. Zeinert, H-J von Bardeleben and R. Bouzerar, Diam. Relat. Mater. 9, 728 (2000).

8 F. Giorgis, F. Giuliani, C. F. Pirri and A. Tagliaferro, Appl. Phys. Lett. 72, 2520 (1998).

9 M.A. Tamor and W.C. Vassell, J. Appl. Phys. 76, 3823 (1994).

10 J.D. Carey and S.R.P. Silva, Phys. Rev. B 70, 235417 (2004).

11 Th. Nguyen-Tran, V. Suendo and P. Roca I Cabarrocas, Appl. Phys. Lett. 87, 0119903 (2005).

12 G. Fanchini and A. Taliaferro, Appl. Phys. Lett. 85, 730 (2004).

13 R. C. Barklie, M. Collins, and S. R. P. Silva, Phys. Rev. B 61, 3546 (2000).

14 J.D. Carey, R.D. Forrest, R.U.A. Khan and S.R.P. Silva, Appl. Phys. Lett. 77, 2006 (2000).

15 A. Abragam, in "Principles of Nuclear Magnetism" p. 126. Clarendon, Oxford, 1996.

16 C. Jäger, J. Gottwald, H. W. Spiess and R. J. Newport, Phys. Rev. B 50, 849 (1994).

17 M. A. Tamor, W.C. Vassell, and K.R. Carduner, Appl. Phys. Lett. 58, 592 (1991). 


\section{Figure captions}

Figure 1 (a) Dispersion of the G peak band position for a polymer-like a-C:H film ( $\mathbf{\square})$, a

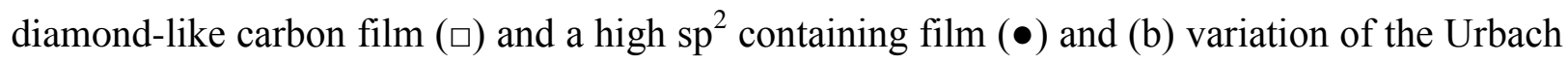
energy with Tauc gap for different a-C:H films.

Figure 2 Variation of (a) spin density and (b) peak-to-peak linewidth for three series of a-C:H films with negative self-bias.

Figure 3 Variation of peak-to-peak linewidth with spin density for the data presented in Figure

2. The dashed line represents the predicted dipolar contribution to the linewidth based on Eq. (1). Note that this is not a fit to data. 

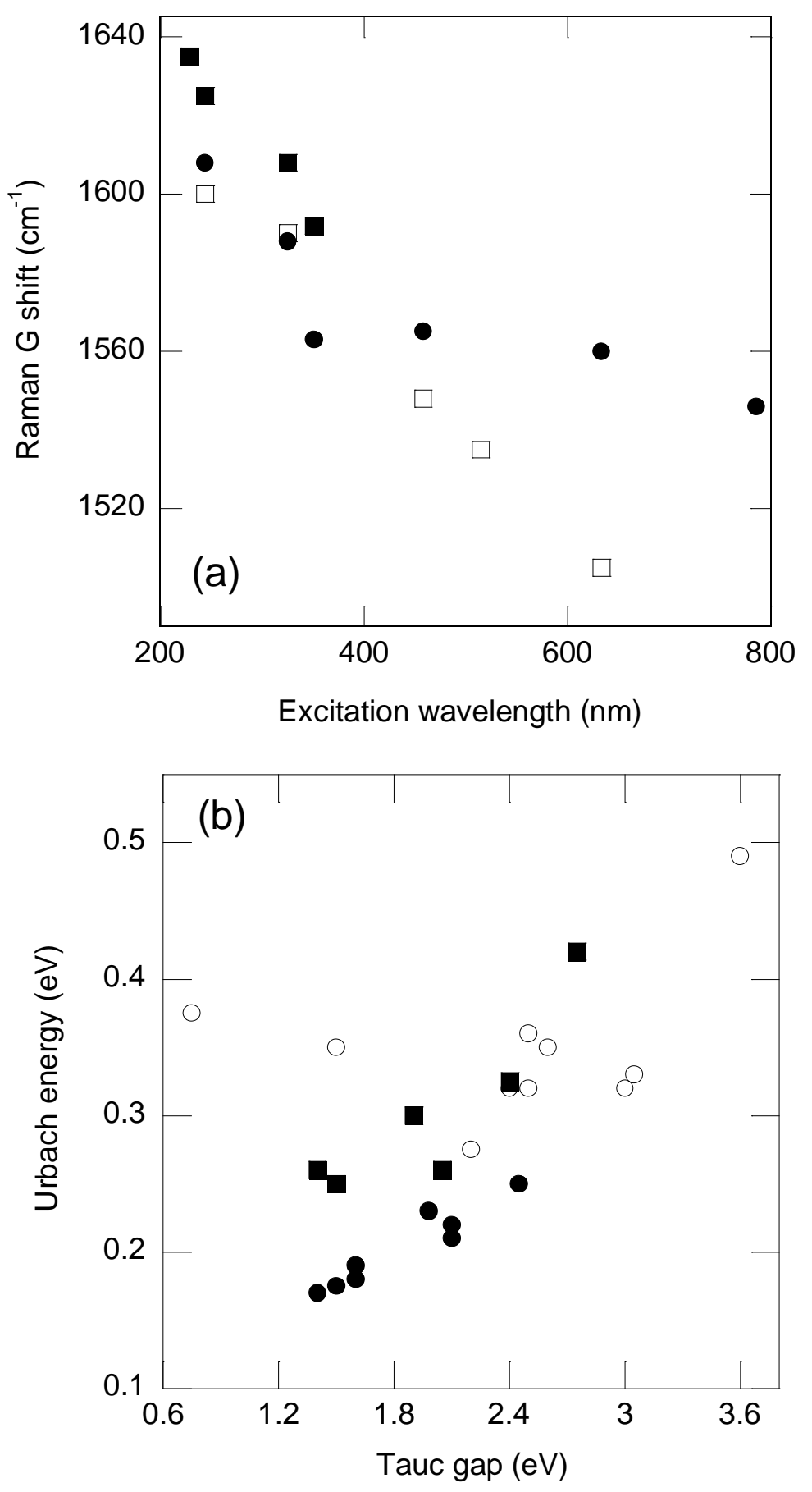

Carey and Silva, Figure 1 

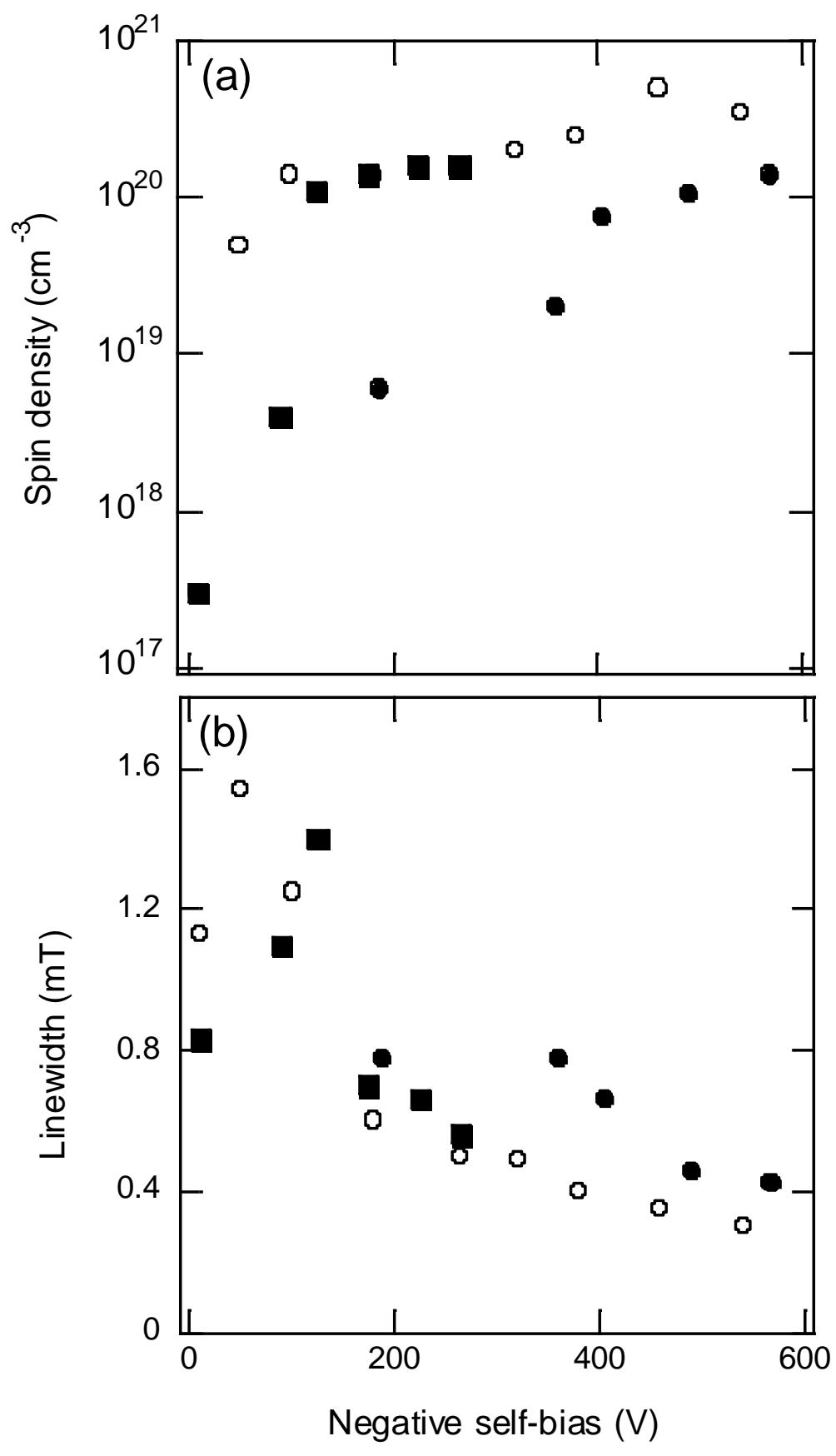

Carey and Silva, Figure 2 


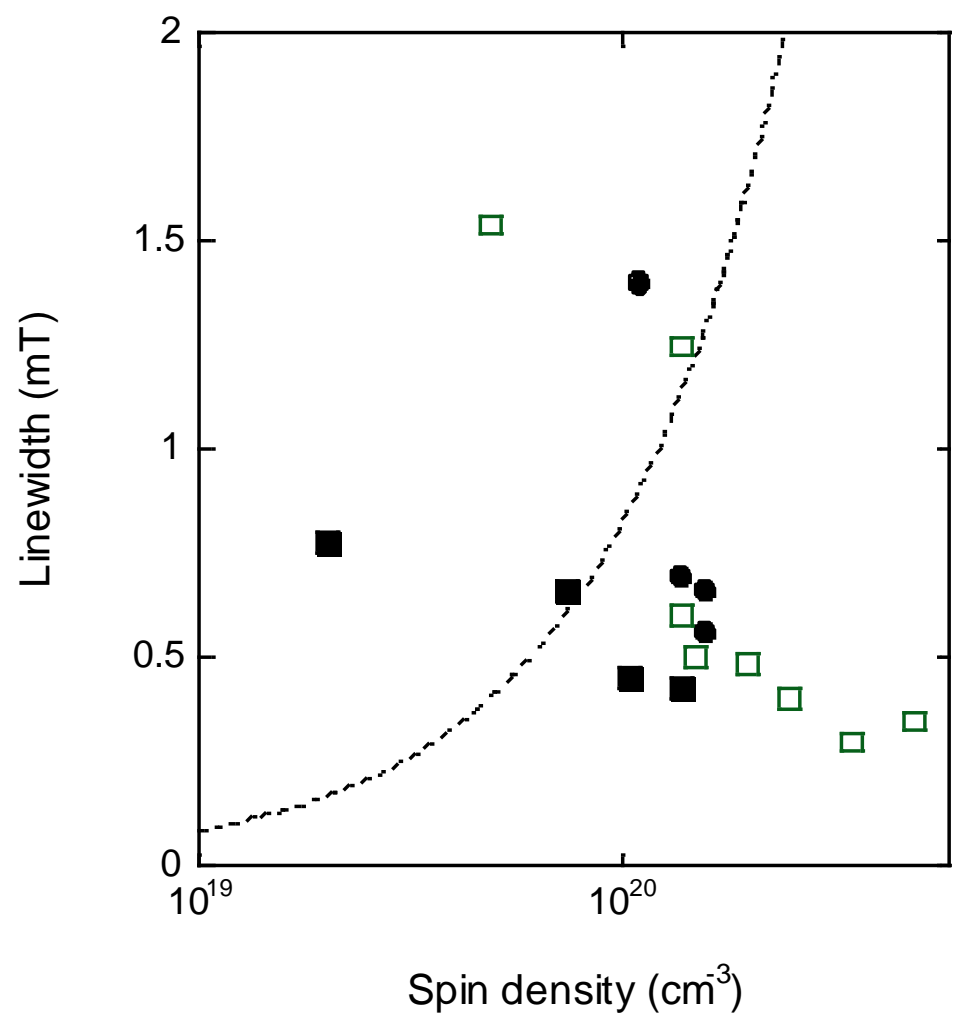

Carey and Silva, Figure 3 